\title{
Influence of Passive OTA Measurement on PCB Antenna
}

\author{
Shuangwen Zhang ${ }^{1, a}$, Zhuoran Zhang ${ }^{2, b}$ and Smart $\mathrm{Li}^{3, \mathrm{c}}$ \\ ${ }^{1}$ Electronic Testing Building,Shahe Road,Xili,Nanshan District,Shenzhen.China \\ 2 Sanyuan Building, No.18 Xibahe Dongli Chaoyang District.Beijing,P.R.China \\ ${ }^{3}$ Electronic Testing Building,Shahe Road,Xili,Nanshan District,Shenzhen.China \\ azhangsw@ccic-set.com, 'bhangzhuoran@ccic.com, Isx@ccic-set.com
}

Keywords: OTA, PCB Antenna, Passive Test.

Abstract. OTA (Over-the-Air) measurement was carried out on a meander PCB antenna for several typical configuration, the passive test results were analyzed and several influence factors for passive OTA test were summarized.

\section{Introduction}

Mobile internet has grown up to be the most popular industry during the past decade [1,2], more and more kinds of smart mobile devices appeared in the markets, as greatly convenienced people's life. Meanwhile, more and more kinds of smart antennas were designed to meet the various requirements [3], especially for the wearable devices [4]. Most of these devices worked at the $2.4 \mathrm{GHz}$ ISM band (Wi-Fi, Bluetooth, etc), and many researchers were concentrating on improving the performance of the antennas, such as MIMO (Multiple Input and Multiple Output) antennas, PCB antennas, etc.

On the other hand, the OTA (Over-the-Air) measurement was an important method to verify the performance of the antenna $[5,6]$. There're two means of OTA measurement, named passive test and active test. Passive test [5] can tell us the parameters such as Pattern, Gain, Efficiency, etc, while active test involves TRP (Total Radiated Power) and TIS (Total Isotropic Sensitivity) [6], they can both check the performance of the design. But for those wireless devices worked at ISM band, active test need more costs, it's always not easy to verify the TRP or TIS, the passive test was a better choice.

In this paper, a passive OTA test was carried out on a meander PCB antenna in several typical configuration, the test results were analyzed.

\section{Configuration of the PCB antenna and Passive OTA Test Setup}

The antenna was designed for a Bluetooth module, meander PCB antenna was selected to comply with the size requirement. The PCB layout in Fig. 1 and Fig. 2 showed the shape of the antenna, the unshielded area of the antenna (from the feedback point to the end of antenna) was $13 \mathrm{~mm} * 5.5 \mathrm{~mm}$.

Fig. 3 showed the preparing for the test, the antenna was connected by a RF cable with SMA connector, different connecting point meant different configuration. Some typical configuration was listed in Table 1:

Table 1: Test configuration of the antenna under test

\begin{tabular}{|c|c|c|}
\hline Configuration & Module shielded or not & Connecting point \\
\hline $1 \#$ & Yes & Feedback point at rear PCB \\
\hline $2 \#$ & No & Feedback point at rear PCB \\
\hline $3 \#$ & Yes & Feedback point at obverse PCB \\
\hline $4 \#$ & No & Feedback point at obverse PCB \\
\hline $5 \#$ & Yes & RF test point at rear PCB \\
\hline $6 \#$ & No & RF test point at rear PCB \\
\hline
\end{tabular}

Fig. 4 was the setup of passive test in an OTA test system including a full anechoic chamber, a vector network analyzer, a turntable and necessary test cable. In this paper, all of the tests were 
executed in the OTA test system with the model TS8991, which was developed by Rohde \& Schwarz, Germany.

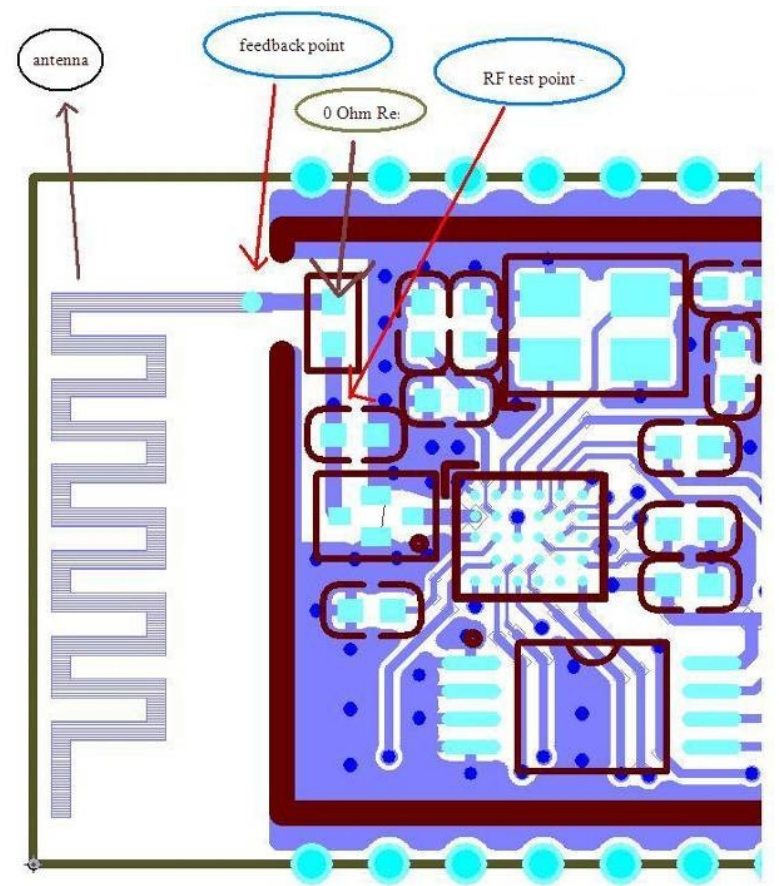

Fig.1 PCB Layout of the antenna (Obverse)

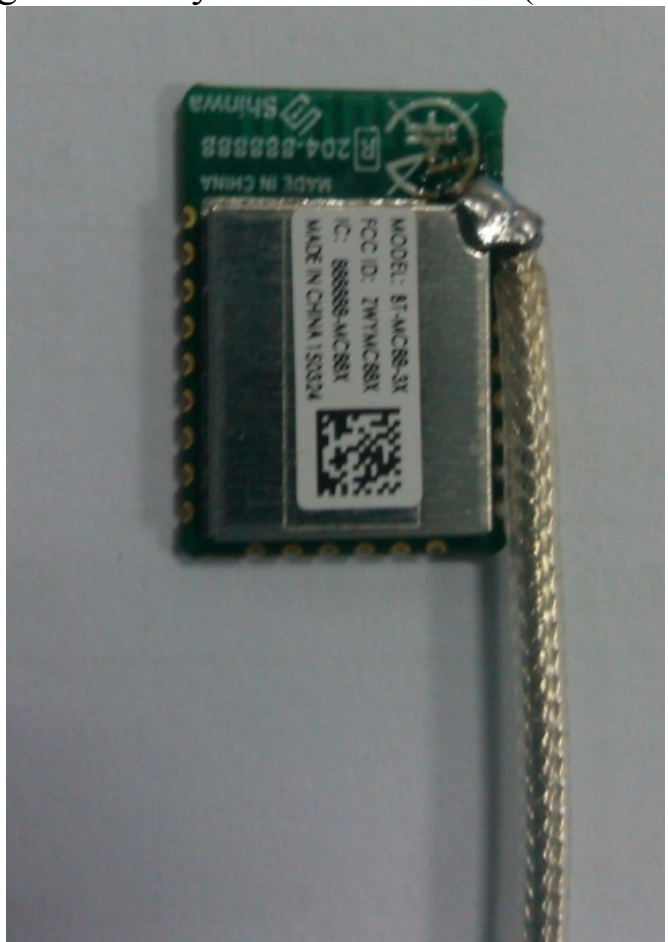

Fig.3 antenna under test

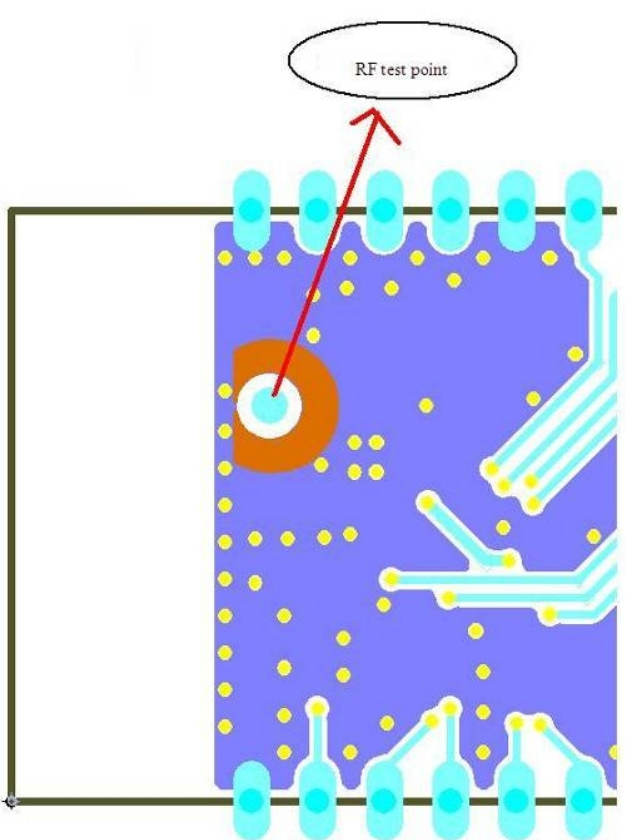

Fig.2 PCB Layout of the antenna (Rear)

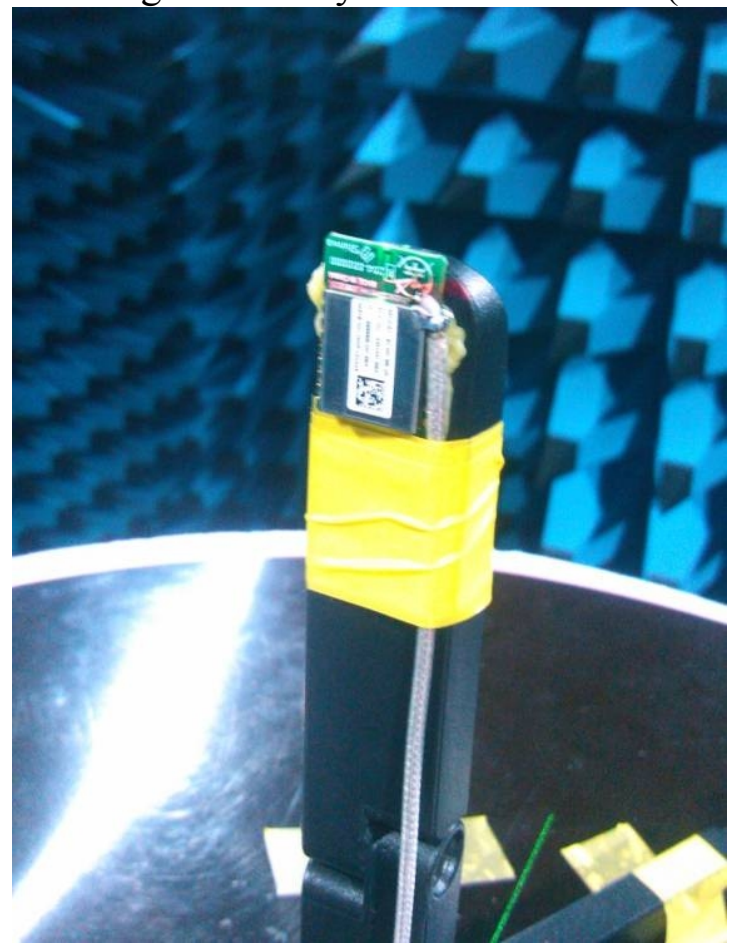

Fig.4 Passive Test Setup

\section{Test Results and Analysis}

The passive tests were carried out at frequency range $2.402 \mathrm{GHz} \sim 2.482 \mathrm{GHz}$, and the results were listed at Table 2 and Fig.5 to Fig.9:

Table 2: Passive test results for different configuration

\begin{tabular}{|c|c|c|}
\hline Configuration & Peak Gain (dBi) & Efficiency (\%) \\
\hline $1 \#$ & 4.28 & 12.3 \\
\hline $2 \#$ & 5.68 & 20.0 \\
\hline
\end{tabular}




\begin{tabular}{|c|c|c|}
\hline $3 \#$ & 4.28 & 15.2 \\
\hline $4 \#$ & 5.56 & 18.98 \\
\hline $5 \#$ & -3.10 & 8.62 \\
\hline $6 \#$ & -5.17 & 4.64 \\
\hline
\end{tabular}

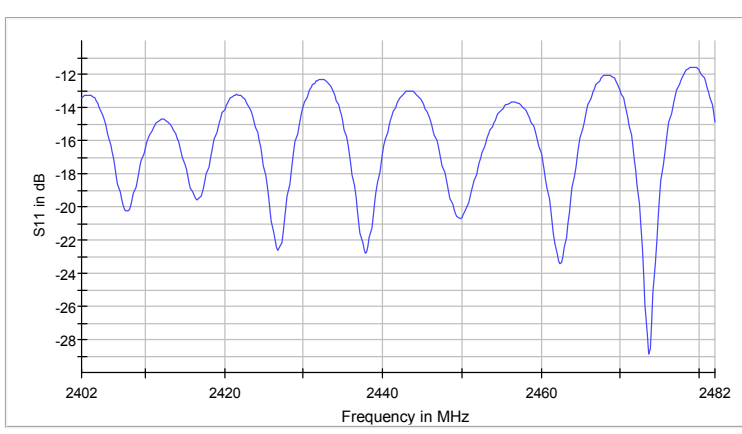

Fig.5 S11 of Configuration 1\#

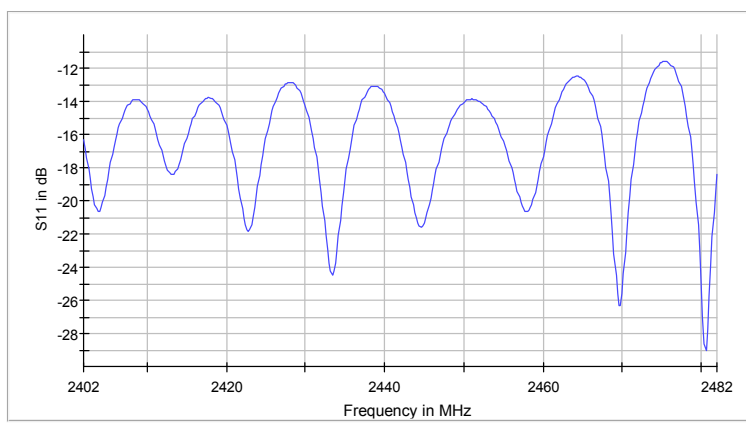

Fig.7 S11 of Configuration 3\#

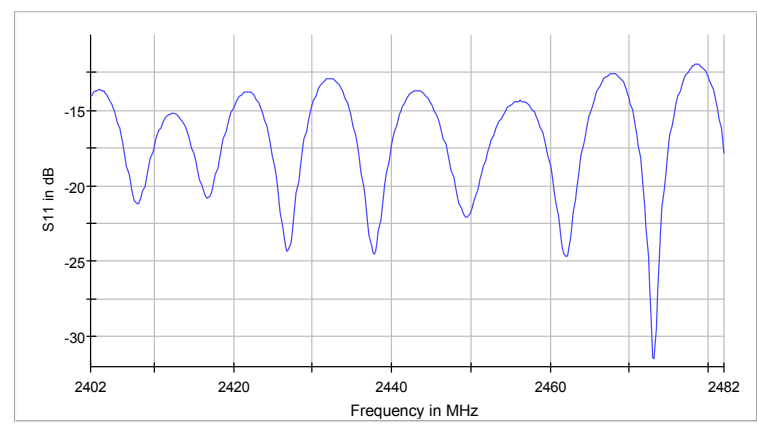

Fig.9 S11 of Configuration 5\#

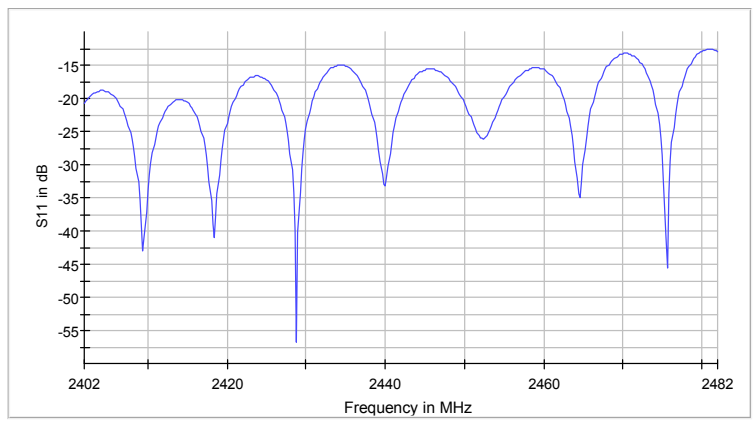

Fig.6 S11 of Configuration 2\#

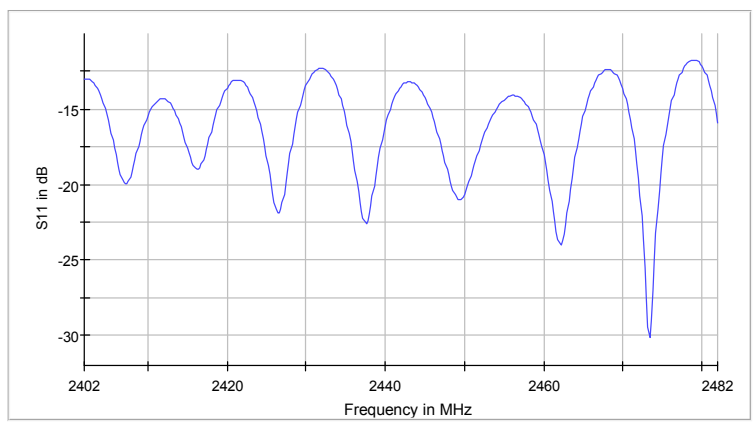

Fig.8 S11 of Configuration 4\#

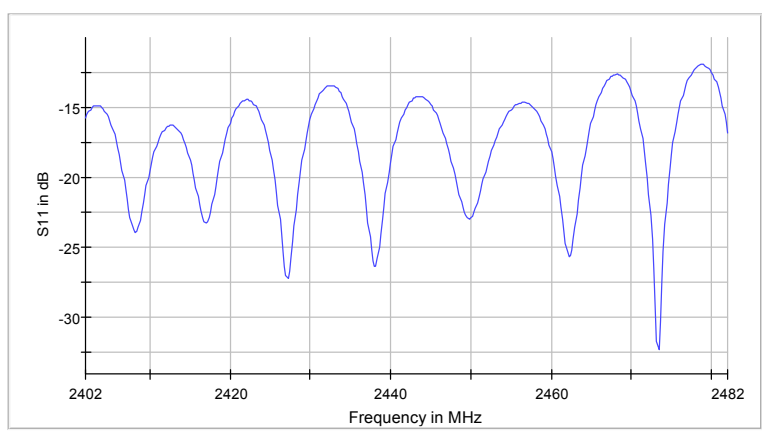

Fig.10 S11 of Configuration 6\#

The results of S11verified the well connection between test points and the RF cable; shielded cover may cause degrading of both the gain and the efficiency; the results of efficiency is not abnormal for this kind of antenna; connecting point could significantly influence the antenna perfomance.

In this case, the RF test point located in the shielded area, the distance between feedback point and RF test point also cause great uncertainty.

\section{Summary}

In this paper, the results of a passive OTA test on a meander PCB antenna were analyzed, the gain and the efficiency will be influenced by connecting point, shielded or not, it may help the antenna designer. 


\section{References}

[1] Information on http://www.gsacom.com

[2] Information on http://www.ctia.org

[3] H. Schantz, Introduction to ultra-wideband antennas, Ultra Wideband System and Technologies, 2003 IEEE Conference (2003) pp. 1-9

[4] Information on http://www.wearabledevices.com

[5] IEEE Standard Test Procedures for Antennas, ANSI/IEEE Std 149-2003

[6] Test Plan for Wireless Device Over-the-Air Performance - Method of Measurement for Radiated RF Power and Receiver Performance, CTIA Certification Test Plan, Ver. 3.4, Washington, DC, December, 2014. 